Rev. High Pressure Sci. Technol., Vol. 7 (1998) $727 \sim 729$

\title{
High-Pressure Raman and X-Ray Scattering Studies on Vermiculite Layered-Silicate Intercalation Compounds
}

\author{
N. Wada and Y. Omura \\ Faculty of Engineering, Toyo University, Kawagoe City, Saitama 350, Japan
}

\begin{abstract}
Single crystals of vermiculite were studied by high-pressure Raman scattering and x-ray diffraction methods using diamond-anvil cells with distilled water and mineral oil as pressure-transmitting media. When water was used, intercalation of water molecules to vermiculite was observed (pressure-induced intercalation). High-pressure Raman spectra from vermiculite samples in various intercalation states revealed the roles of interlamellar intercalant molecules and atoms in the lattice dynamics of vermiculite.

[vermiculite, intercalation, Raman scattering, high pressure, $x$-ray diffraction]
\end{abstract}

\section{Introduction}

Layered silicates exhibit intriguing physical and chemical properties because of their highly anisotropic structure. In particular, intercalation of atoms and molecules to expandable layered silicates is of great interest, since intercalation causes drastic changes in the structure and thus their physical properties. Application of pillared clays (in which large intercalant molecules expand the basal spacing to provide more space) for catalysis is noteworthy [1].

Recently it was shown that vermiculite, one of the 2:1 layered silicates, can be intercalated with alkali-metal atoms by vapor transport [2]; transparent vermiculite crystals exhibit a metallic silver color and the electrical conductivity increases drastically upon intercalation (a quasi-2D conductor).

Applying high pressure is an effective method to study layered-silicate intercalation compounds, especially because the intralayer interactions are much stronger relative to the interlayer interactions. Structural and lattice-dynamical studies will certainly benefit from high-pressure experiments.

Here, we discuss the structure and lattice dynamics of vermiculite in two extreme cases; one is pressure-induced water-molecule intercalation and the other is alkali-metal intercalation. Pressure and intercalant dependence of the structure and the host-layer phonon modes are discussed.

\section{Experimental}

Single-crystal Llano vermiculite was used for our experiments. The approximate chemical composition[3] is $\left(\mathrm{Si}_{5.72} \mathrm{Al}_{2.28}\right)$ $\left(\mathrm{Mg}_{5.58} \mathrm{Al}_{0.10} \mathrm{Fe}_{0.03} \mathrm{Ti}_{0.02}\right) \mathrm{O}_{20}(\mathrm{OH})_{4} \mathrm{Mg}_{0.93} \cdot m\left(\mathrm{H}_{2} \mathrm{O}\right)$, where the number of water molecules $m$ depends on its hydration states [4]. The samples were first cation-exchanged by immersing in salt solutions for months; interlamellar $\mathrm{Mg}$ cations were replaced with desired alkali-metal cations. The completion of cation exchange was confirmed by $\left(\begin{array}{lll}0 & 0 & \ell\end{array}\right) x$-ray diffraction patterns. Vapor-transport intercalation of alkali metals to vermiculite was carried out using a two-temperature-zone-furnace method[2].

Diamond-anvil cells were used for both high-pressure $\mathrm{x}$-ray and Raman scattering experiments. The pressure-transmitting media were distilled water and mineral oil (as described below, water molecules were found to intercalate $\mathrm{Na}$ vermiculite, while no evidence for oil-molecule intercalation was found in the mineral oil case). The pressure calibration was done by measuring the ruby-fluorescence wavelength shift with pressure.

$\mathrm{X}$-ray diffraction was conducted using a Huber four-circle diffractometer with a conventional Mo x-ray tube. Raman scattering was done using a Spex monochromator and an Ar-ion laser $(\lambda=5145 \AA)$.

\section{Results and Discussions}

Single-crystal Na vermiculite was first studied in distilled water under pressure up to $25 \mathrm{kbar}$. Even at ambient pressure, the basal spacing of Na vermiculite $(\mathrm{d}=14.95 \AA)$ was found to be larger than that in air $(\mathrm{d}=14.89 \AA)$. This fact implies that there may exist more water molecules in the interlamellar space of $\mathrm{Na}$ vermiculite in bulk water. Figure 1 shows $\mathrm{x}$-ray diffraction patterns taken at 3.5 and $13 \mathrm{kbar}$. The insert shows the pressure dependence of the $(001)$ peak. The observation of the $(00 \ell)$ peak positions as a function of pressure suggests that the basal spacing of Na vermiculite first expands and then contracts with increasing pressure. The expansion may be caused by pressure-induced intercalation of water molecules. Also one can notice that there is a small peak at $\mathrm{q}=0.394 \AA^{-1}$, which might correspond to a new hydration state with $\mathrm{d}=15.95 \AA$. More detailed discussions on the pressure-induced intercalation will be found elsewhere[5].

Figs. 2 and 3 show pressure-dependent Raman spectra taken from $\mathrm{Na}$ vermiculite immersed in distilled water in a diamondanvil cell at room temperature. The pronounced peak at $\sim 155$ $\mathrm{cm}^{-1}$ was assigned as the $\mathrm{A}^{\prime}$ mode that involved vibrations of surface oxygen atoms along the $\mathrm{c}$ axis[6]. By simply immersing $\mathrm{Na}$ vermiculite in bulk water, the frequency of the $A^{\prime}$ mode was found 


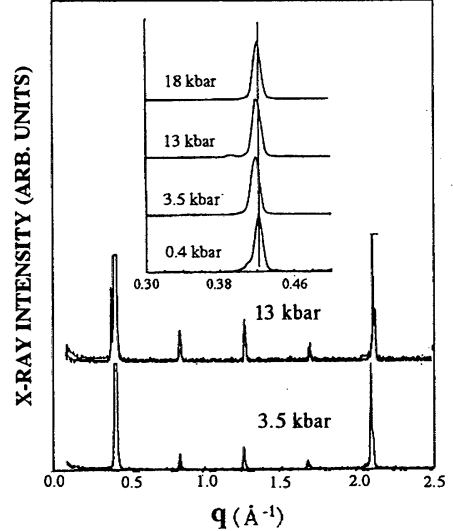

Fig. 1. $(00 \ell) x$-ray diffraction patterns taken from Na vermiculite in water (as a pressure transmitting medium) at 3.5 and 13 kbar at room temperature. The insert shows expanded $x$-ray diffraction patterns of the (001) peak. The vertical line is a guide for your eye.

to down-shift by $\sim 10 \mathrm{~cm}^{-1}$, implying that more water molecules were introduced to the interlamellar space (consistent with the $x$ ray result mentioned above). The width of the A' peak became narrower by immersing in bulk water; the width of the peak changed from $\sim 22 \mathrm{~cm}^{-1}$ to $\sim 12 \mathrm{~cm}^{-1}$, close to half the value. The Raman peak is quite sensitive to the interlamellar water molecules.

The peak frequency and width of the A' mode are plotted in Fig. 4. With increasing pressure, the peak frequency appeared to shift linearly, while the width did not change much with pressure. The least-square fitted slope yields $\mathrm{d} \omega / \mathrm{dp} \sim 0.52 \mathrm{~cm}^{-1} / \mathrm{kbar}$ for the $A^{\prime}$ mode. In contrast, the Raman peak at $677 \mathrm{~cm}^{-1}$ (probably corresponding to a vibration of $\mathrm{Mg}$-O groups in the octahedral layer) shifted with $\mathrm{d} \omega / \mathrm{dp} \sim 0.69 \mathrm{~cm}^{-1} / \mathrm{kbar}$. Considering the original frequencies, the $A^{\prime}$ mode changed more drastically with in-
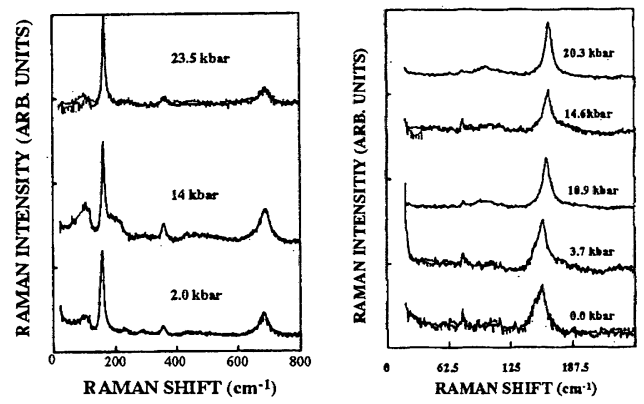

Figs. 2 and 3. Raman spectra taken from Na vermiculite in distilled water (as a pressure-transmitting medium) at different pressures.

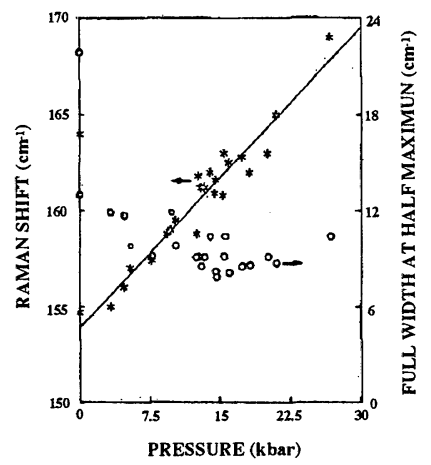

Fig. 4. Pressure dependence of the $A^{\prime}$-mode Raman peak of $\mathrm{Na}$ vermiculite in distilled water. The data points for the Raman shift are indicated by *, whereas those for the FWHM are indicated by 0 .

creasing pressure.

In addition, we mention about the other extreme case where the interlamellar water molecules are completely taken out from Na vermiculite. In dehydrated Na vermiculite $(d=9.62 \AA)$, the $A^{\prime}$ peak was found to be $\sim 194 \mathrm{~cm}^{-1}$ that is about $20 \mathrm{~cm}^{-1}$ higher in frequency than $\mathrm{Na}$ vermiculite in two-water-layer hydration state ( $\mathrm{d}=14.89 \AA)$.

Fig. 5 shows Raman spectra taken from $\mathrm{K}$ cation-exchanged and $\mathrm{K}$ doped vermiculites. The "K cat-ex Vermiculite (as-is)" spectrum was taken from $\mathrm{K}$ cation-exchanged vermiculite in air at ambient conditions. When completely dehydrated ("K cat-ex Vermiculite (dry)), a drastic increase in the amount of luminescence from the sample was observed. Both the $A^{\prime}$ peak and $683-\mathrm{cm}^{-1}$ peak appeared to have down-shifted somewhat after driving out the interlamellar water molecules (the $d$ spacing of "as-is" sample was slightly larger than that of "dry" sample, implying that there existed a small amount of interlamellar water molecules in the "as-is" sample). The " $K$ doped Vermiculite" spectrum was taken from a vermiculite sample prepared by the vapor-transport inter-

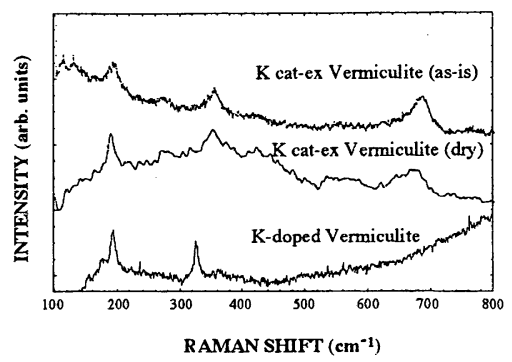

Fig. 5. Raman spectra taken from $\mathrm{K}$ cation-exchanged and $\mathrm{K}$ doped vermiculites. Scattering from the glass ampule is subtracted. 
calation method mentioned above. Our $\mathrm{x}$-ray diffraction experiments showed that the basal spacing for $\mathrm{K}$ vermiculite became slightly shorter upon intercalation, possibly because some charge transfer might have occurred from the guest atoms to the host layer[2]. As seen in Fig. 5, significant changes were observed in the $\mathrm{K}$-dope-vermiculite Raman spectrum in comparison with the "dry" spectrum. Upon K-atom intercalation, (1) the A' peak frequency became $\sim 193 \mathrm{~cm}^{-1}$, that was higher by $\sim 3 \mathrm{~cm}^{-1}$ than that of "dry sample. (2) the Raman peak at $\sim 360 \mathrm{~cm}^{-1}$ downshifted by $35 \mathrm{~cm}^{-1}$, (3) both the A' peak at $193 \mathrm{~cm}^{-1}$ and the Raman peak at $360 \mathrm{~cm}^{-1}$ became narrower in width, (4) the peak at $\sim 680 \mathrm{~cm}^{-1}$ seemed to have disappeared.

We speculate that upon intercalation $\mathrm{K}$ intercalant atoms may occupy empty pseudo-hexagonal holes in the silicate layers (in our samples, the amount of unoccupied holes are estimated to be $\sim 7 \%$ when "dried", considering the chemical composition of Llano vermiculite mentioned above). Then, the crystallinity of intercalated vermiculite might improve once having more interlamellar intercalant atoms in the holes. This speculation supports the fact that the Raman peak widths became narrower upon intercalation. The reason why the $680 \mathrm{~cm}^{-1}$ peak disappeared upon intercalation might be related to a change in the Raman selection rules caused by the crystal-structure change.

Fig. 6 shows Raman spectra taken from dehydrated $\mathrm{K}$ cationexchanged vermiculite as a function of pressure up to $38.7 \mathrm{kbar}$ at room temperature. Here, the pressure-transmitting medium was mineral oil. The A' peak frequency appears to have shifted linearly with pressure. The rate of frequency shift with increasing pressure can be found by plotting the peak-frequency vs. pressure, as shown in Fig. 7. The rate d $\mathrm{d} / \mathrm{dp}$ for the $\mathrm{A}^{\prime}$ peak of $\mathrm{K}$ cation-exchanged vermiculite was found to be $\sim 0.19 \mathrm{~cm}^{-1} / \mathrm{kbar}$, which is much smaller than that of water-molecule intercalated $\mathrm{Na}$ ver-

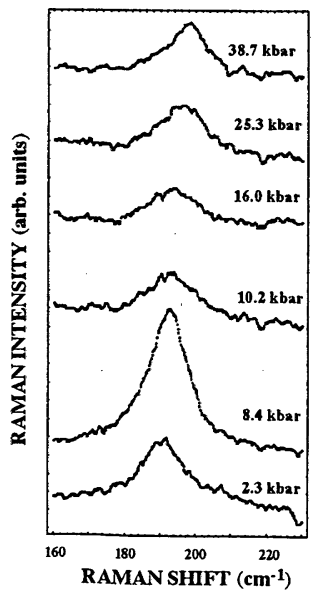

Fig. 6. Pressure dependent Raman spectra taken from K cationexchanged vermiculite at room temperature. The pressure transmitting medium was mineral oil.

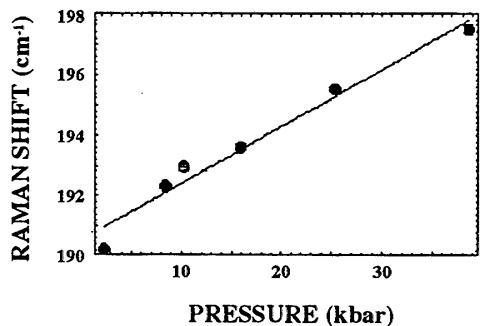

Fig. 7. Pressure dependence of the $A^{\prime}$-mode Raman peak of $K$ cation-exchanged vermiculite. The straight line indicates the best least-square fit.

miculite $\left(\sim 0.52 \mathrm{~cm}^{-1} / \mathrm{kbar}\right)$. The surface oxygen atoms of the tetrahedral layers may have hydrogen bonds with interlamellar water molecules in the pressure-induced intercalation case, and thus the nature of the vibration may differ significantly.

\section{Concluding Remarks}

It was shown that pressure-induced intercalation could occur if bulk water was chosen as a pressure-transmitting medium. Also, it was demonstrated that the inplane intercalant structure significantly affected the lattice dynamics of vermiculite. In particular, the A' mode (vibration of the surface oxygen atoms) were found to be quite sensitive to intercalant species and applied pressure.

It can be mentioned that the layered-silicate system are quite attractive for fundamental research because one can observe drastic effects on physical properties when pressure is applied. Various intercalation processes promise more diverse research opportunities. Especially, alkali-metal vapor-phase intercalation, which causes a drastic increase in conductivity, is of our current central interest.

\section{Acknowledgments}

The authors thank D.R. Hines and T. Takeishi for technical assistance. The work was supported by the special research funds from Toyo University. Y.O. is especially grateful to the Enryo Inoue Fellowship from Toyo University.

\section{References}

[1] I.V. Mitchell (ed.), Pillared Layered Structures:Current Trends and Applications, Elsevier, London (1990).

[2] N. Wada, H. Okui, Y. Omura, A. Fujiwara, H. Suematsu, and Y. Murakami, Mater. Res. Soc. Symp. Proc. 453, 95 (1997).

[3] P.G. Slade, C. Dean, P.K. Schultz, and P,G. Self, Clays Clay Miner. 35, 177 (1987).

[4] M. Suzuki, N. Wada, D.R. Hines and M.S. Whittingham, Phys. Rev. B 36, 2844 (1987).

[5] N.Wada and D.R. Hines, to be published.

[6] N. Wada, M. Suzuki, D.R. Hines, K.Koga and H. Nishihara, J. Mater. Res. 2, 864 (1987). 